\title{
Reverse Total Shoulder Replacement
}

\section{Intraoperative and Early Postoperative Complications}

\author{
Carl Wierks MD, Richard L. Skolasky ScD, \\ Jong Hun Ji MD, Edward G. McFarland MD
}

Received: 1 November 2007/Accepted: 7 July 2008/Published online: 7 August 2008

(C) The Association of Bone and Joint Surgeons 2008

\begin{abstract}
Reverse total shoulder arthroplasty is a treatment option for patients with symptomatic glenohumeral arthritis and a deficient rotator cuff. The reported complication rates vary from $0 \%$ to $68 \%$. Given this variation, our purposes were to (1) determine the learning curve for the procedure, (2) identify complications and surgical pitfalls, and (3) compare our results with those of similar published series. We retrospectively reviewed 20 consecutive patients (mean age, 73 years; range, $45-88$ years) who had reverse total shoulder arthroplasty by one surgeon, tabulating intraoperative and postoperative complications. Minimum followup was 3 months (average, 9 months; range, 3-21 months). The intraoperative complication rate for the first 10 patients was higher than that for the second 10 patients. There were 33 complications in 15 patients: 11 patients collectively had 22 intraoperative complications and eight patients collectively had 11 postoperative complications. At radiographic
\end{abstract}

Each author certifies that he or she has no commercial associations (eg consultancies, stock ownership, equity interest, patent/licensing arrangements, etc.) that might pose a conflict of interest in connection with the submitted article.

Each author certifies that his or her institution has approved the human protocol for this investigation, that all investigations were conducted in conformity with ethical principles of research, and that informed consent was obtained.

C. Wierks, R. L. Skolasky, J. H. Ji, E. G. McFarland Division of Sports Medicine and Shoulder Surgery, Department of Orthopaedic Surgery, Johns Hopkins University, Baltimore, MD, USA

E. G. McFarland ( $\square)$

c/o Elaine P. Henze, BJ, ELS, Medical Editor, Department of Orthopaedic Surgery, Johns Hopkins Bayview Medical Center, 4940 Eastern Ave., \#A672, Baltimore, MD 21224, USA

e-mail: ehenze1@jhmi.edu followup, 11 patients had scapular notching and nine patients had heterotopic ossification. Our complication rate was higher than published rates.

Level of Evidence: Level II, prognostic study. See the Guidelines for Authors for a complete description of levels of evidence.

\section{Introduction}

Reverse shoulder arthroplasty was described first by Grammont et al. [10] in 1987 as a treatment for patients with cuff tear arthropathy for which nonoperative modalities had failed. Other indications for prosthesis implantation include revision of a failed arthroplasty or internal fixation of a proximal humerus fracture associated with a rotator cuff deficiency [1], reconstruction after tumor resection of the proximal humerus $[4,5]$, and pseudoparalysis of the shoulder [30]. Requirements for implantation include a functional deltoid muscle and adequate glenoid and proximal humeral bone quality [8].

Although reverse total shoulder arthroplasty has been performed for more than 20 years in Europe with promising results $[2,6,11,21,22,25,28]$, it was not approved for use in the United States until March 2004. Since that time, numerous outcomes of the reverse prosthesis have been published $[1,5,9,12,26,29,30]$. The reported complication rate for this procedure ranges from $0 \%$ to $68 \%$ (Table 1), but these studies have not focused on intraoperative complications of this procedure nor emphasized pitfalls in technique during early experience of the surgeons. Because of these factors, one surgeon suggested reverse prosthesis surgery should be performed only by experienced shoulder surgeons [23]. 


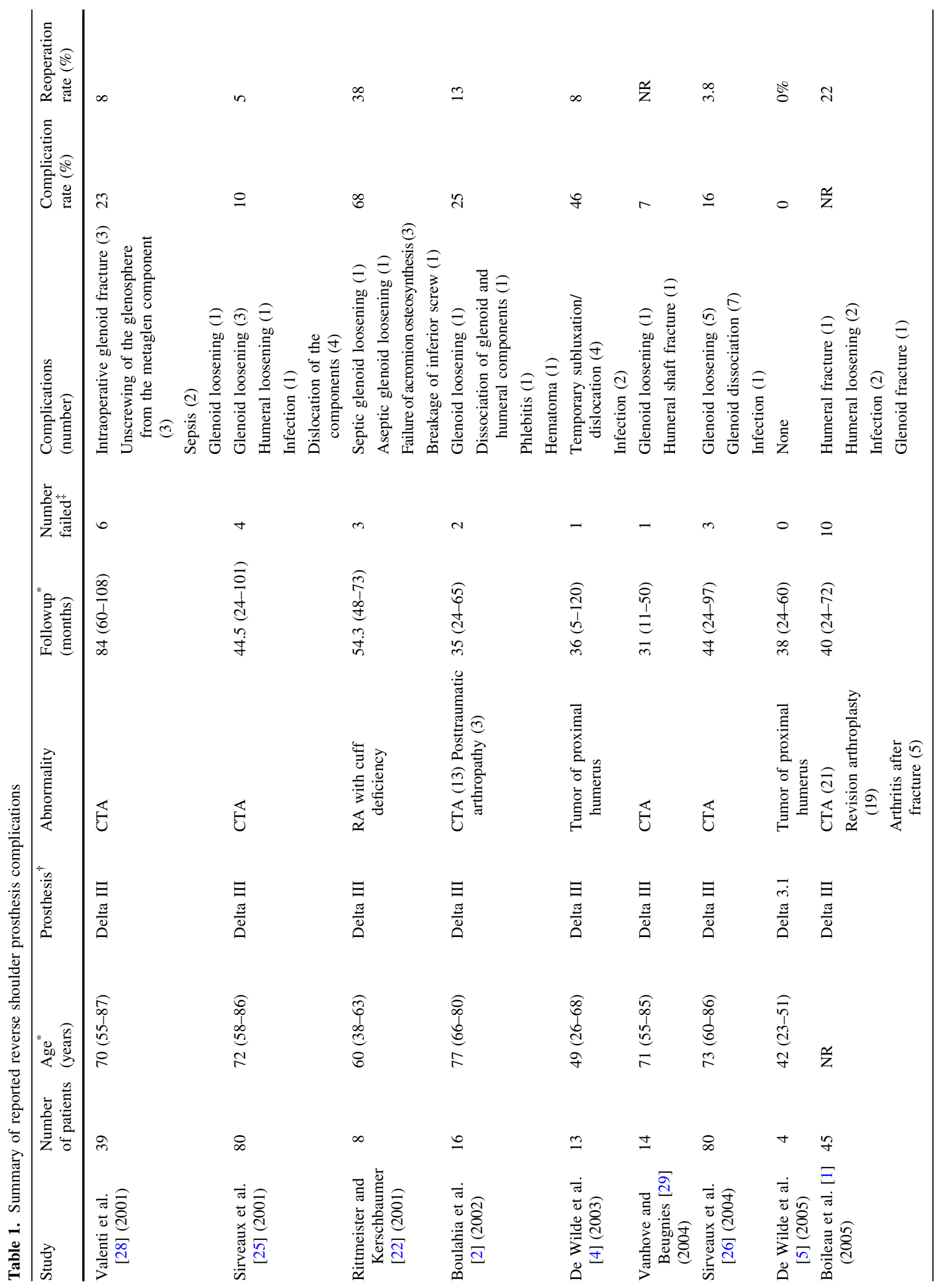




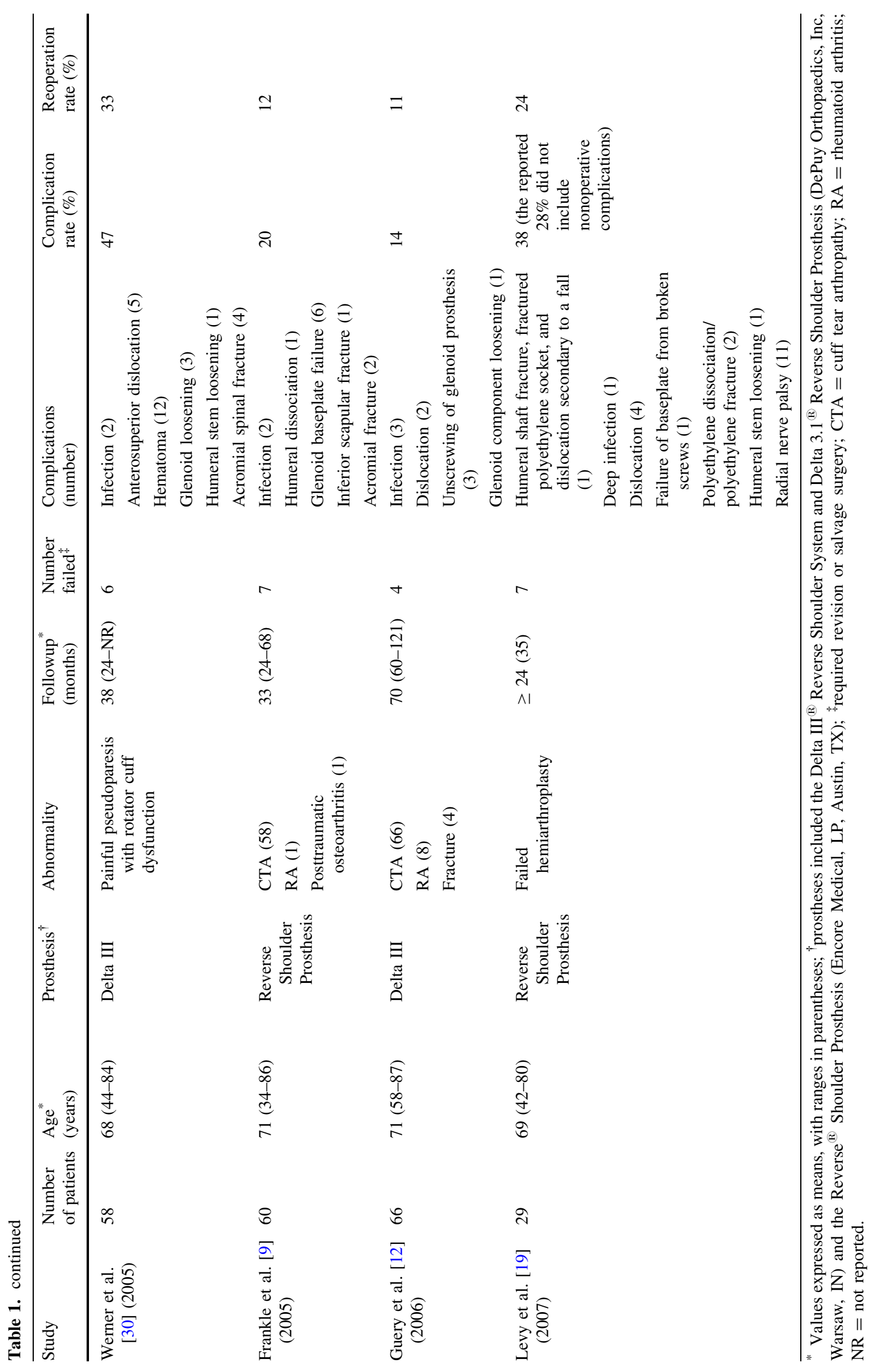


Our hypotheses were: (1) our observed complication and pitfall rates of this surgical procedure would be similar to published rates, and (2) the intraoperative and postoperative complication rates would be higher among early procedures than in later procedures. We also sought to determine if there was a definable learning curve for this procedure after which the late complication rate decreased markedly from the early complication rate. We sought to determine if preoperative patient demographics and clinical features were associated with an increased risk of intraoperative and postoperative complications. Finally, because we found several radiographic complications not previously reported (such as heterotopic ossification), we sought to determine the incidence of complications detectable by radiographs in patients undergoing a reverse prosthesis.

\section{Materials and Methods}

We retrospectively reviewed the charts of 20 consecutive patients who had a reverse total shoulder arthroplasty performed by the same surgeon between June 2004 and May 2006 and who had at least 3 months followup. Patients with surgery after this time were not included because they had less than 3 months followup. Key variables extracted included patient demographics (gender and age), indications for surgery, presence of intraoperative complications and surgical pitfalls, and postoperative complications. A formal power analysis was not conducted. A review of the literature suggested the learning curve for most surgical procedures was approximately 10 cases $[3,7]$. Therefore, we divided the 20 patients into two groups: the first 10 patients and the second 10 patients. Key patient demographics included gender and age. The age of the 16 female and four male patients averaged 73 years (range, 44-88 years). The minimum followup was 3 months (average, 9 months; range, 3-21 months). No patient was seen for followup specifically for this review. This study was reviewed and approved by our Institutional Review Board.

The indications for surgery were symptomatic glenohumeral arthritis and massive rotator cuff deficiency (16 patients) or pain after hemiarthroplasty (four patients; cuff tear arthropathy $[\mathrm{n}=2]$, painful anterior-superior subluxation syndrome $[\mathrm{n}=2]$ ) for which nonoperative treatment had failed. The preoperative diagnosis was based on the clinical examination and radiographs showing decreased acromiohumeral space. Not all patients had MRI because of the presence of an implant. In the patients with MRI $(n=15)$ or computed tomography arthrography $(n=1)$, there was a complete tear of the supraspinatus muscle (16 patients), associated tears of the infraspinatus muscle alone (three patients), tears of the subscapularis muscle alone (two patients), or tears of the infraspinatus and subscapularis muscles (seven patients). Fourteen patients had had previous surgeries on the operative shoulder, 13 had had rotator cuff repairs, and nine had had multiple surgeries.

All surgeries were performed by the senior author (EGM), whose practice is $90 \%$ shoulder surgeries, including approximately 50 shoulder arthroplasties per year. For the first four patients, the DePuy reverse shoulder prosthesis (DePuy Orthopaedics, Inc, Warsaw, IN) (Fig. 1) was used; for the subsequent 16 patients, the Tornier reverse shoulder prosthesis (Tornier Inc, Stafford, TX) (Fig. 2) was used. The designs of and instrumentations for these two systems are similar.

The surgery was performed with the patients in the beach chair position. In every case, general anesthesia with

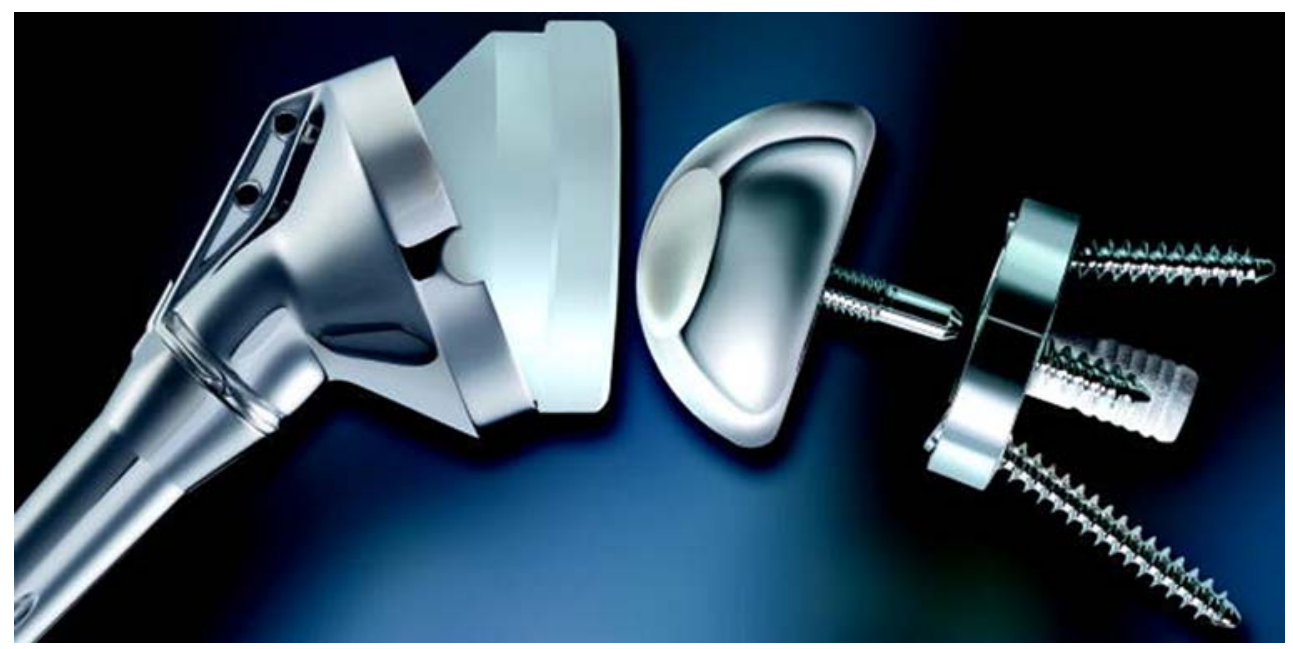

Fig. 1 A diagram illustrates the DePuy reverse shoulder prosthesis (Courtesy of DePuy Orthopaedics, Inc, Warsaw, IN). 


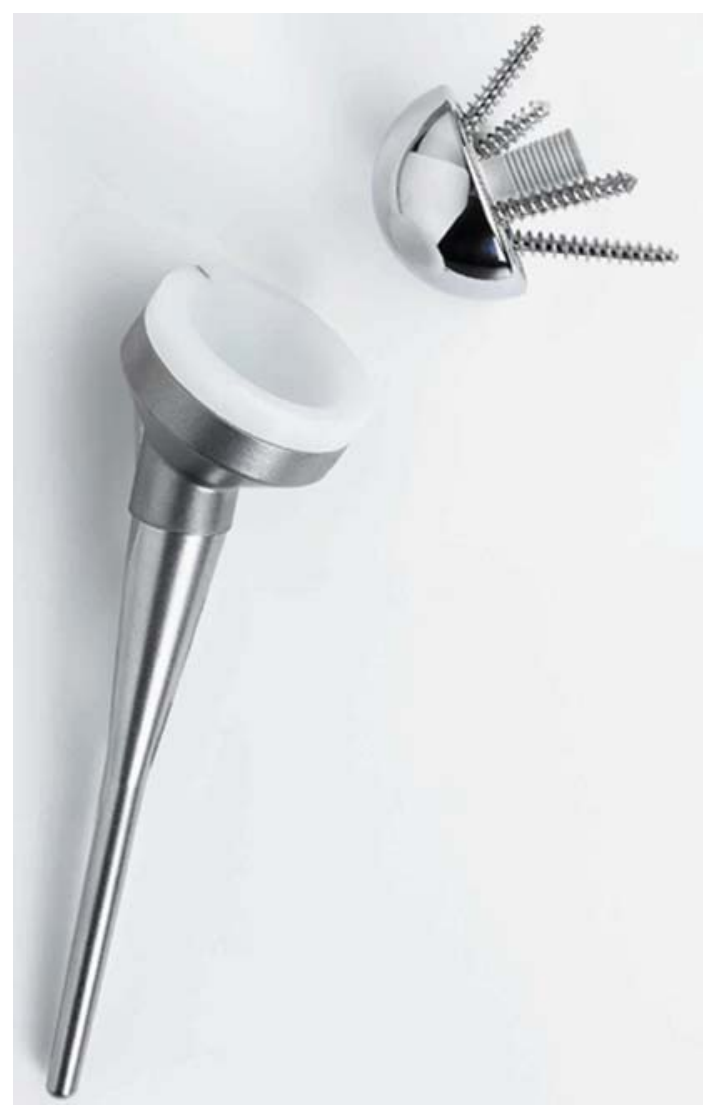

Fig. 2 A photograph shows the Tornier reverse shoulder prosthesis (Courtesy of Tornier Inc, Stafford, TX).

a scalene block or indwelling scalene catheter was used, and perioperative antibiotics were administered. A deltopectoral incision was used, and the cephalic vein was protected when possible. The deltoid was released carefully from the underlying structures, and the upper $1 \mathrm{~cm}$ of the pectoralis major tendon was released in patients with an internal rotation contracture. The joint capsule was split in line with the bicipital groove and extended into the rotator cuff interval. If the biceps tendon was present, it was released, and a tenodesis to the anterior capsule was performed at the end of the procedure in every case.

Exposure of the proximal humerus was attained by creating anterior and posterior periosteal flaps, removing osteophytes, and circumferentially releasing the capsule. The humeral head osteotomy was made between $10^{\circ}$ and $15^{\circ}$ retroversion. For the first three patients, the proximal humerus was prepared before inserting the glenoid baseplate, but because of a high number of rim fractures in the proximal humerus with this sequence, the glenoid component was inserted first in subsequent patients, followed by preparation of the proximal humerus and insertions of the humeral component.

Exposure of the glenoid was obtained with anterior and posterior retractors. Particular attention was paid to exposing and identifying the inferior column of bone in the lower scapula. The glenoid was sized, and for the initial 16 patients, the centering hole was made through a guide. However, difficulty in keeping the guide in place on the smooth surface of the glenoid resulted in the centering hole in an unusable location. Therefore, a small anchoring hole was made in the glenoid for the center peg reamer, a hole that prevented drill migration. As a result, after 16 patients, hole drilling for the baseplate central peg was performed by hand without a guide after creating a small anchoring hole.

Next, the glenoid was reamed with a reamer of appropriate size for the baseplate. Initially reaming was performed with a pneumatic power drill, but its high torque resulted in two nondisplaced fractures of the glenoid (from the glenoid anchoring hole through the anterior rim of the glenoid). In both cases, the baseplate had what was judged to be adequate bone superiorly and inferiorly to support the component. However, in both cases, cancellous bone from the patient's humeral head was placed in the central peg hole and anterior to the glenoid along the scapular neck. Subsequent to the second fracture, the glenoid was reamed by hand. No other fractures occurred. The centering hole then was over-reamed and the baseplate was impacted into place.

To allow compression from the anterior and posterior screws, the superior and inferior screws were placed into the baseplate first but were not locked. The anterior and posterior screws then were placed; we recorded whether compression was obtained or the screws spun. In three patients, no screw was placed anteriorly because of insufficient bone stock. In the remaining patients, the screws were left in place regardless of whether we obtained solid fixation, in hopes they might provide stability by soft tissue or bone formation around the screw. Once the baseplate screws were in place, the glenoid sphere was inserted onto the baseplate and locked into place with the central screw. We defined inadequate screw fixation as inability to place a compression screw or the lack of compression provided by a compression screw. For these systems, four-screw fixation was considered optimum, and we defined suboptimum fixation as anything less than adequate four-screw fixation.

The proximal humerus was prepared by proximal reaming of the humeral head. During the humeral shaft sizing, care was taken to avoid penetrating the humeral shaft. A trial reduction with appropriately sized components was performed, and the soft tissue tension of the joint was assessed. The arm was tested for positions in which the implant might subluxate or dislocate, and an axial distraction force was applied to ensure component stability. Finally, the definitive humeral implant was cemented into place with a cement restrictor distal to the stem.

Associated procedures at the time of surgery included biceps tenodesis (six patients), glenoid bone grafting (four 
patients), and use of Dall-Miles cables (Stryker-Howmedica, Kalamazoo, MI; two patients). Bone grafting (autologous humeral head bone [three patients], freezedried bone chips [one patient]) was performed for anterior glenoid erosion (two patients) and to augment an iatrogenic glenoid rim fracture (two patients). Dall-Miles cables were placed to support a humeral bone window that was created to facilitate hardware extraction in one patient and to augment repair of a calcar fracture in another.

The wound was closed over two drains, and the patient was placed in a commercially available shoulder immobilizer. Passive range of motion (ROM) of the elbow and active and passive ROM of the wrist and hand were permitted the next day. The drains were removed on postoperative Day 1 for all patients. All patients received at least 24 hours of prophylactic antibiotics.

All patients were hospitalized for at least 2 days postoperatively and then seen in clinic 1 week after surgery, at which time the shoulder immobilizer was replaced with a sling. Patients were allowed to remove the sling for ROM elbow exercises and pendulum exercises. No patient had formal physical therapy until 3 months after surgery.

Patients routinely were seen on a monthly basis for 3 months and then yearly thereafter. Some patients initiated more frequent followup. At each followup, the treating surgeon (EGM) did a complete shoulder examination, including a neurovascular examination of the upper extremity. We obtained radiographs (axillary view and anteroposterior views with the arm in internal and external rotation) of the operative extremity. One author (EGM) interpreted all radiographs. Scapular notching was graded according to the system of Nerot as described by Valenti et al. [28] (Fig. 3). Any heterotopic ossification was recorded and graded via the classification of KjaersgaardAndersen et al. [15]. Our last radiographs were taken a minimum of 3 months after surgery (mean, 9 months; range, 3-21 months).

To test for differences between continuous variables (eg, age) in the first 10 and second 10 procedures, we used Student's t test, and to test for group differences between categorical variables (eg, gender), the chi square test of association for categorical measures was used. We checked the normality assumption using the Shapiro-Wilk test. We observed no difference between the two groups regarding age, gender, or history of previous surgeries of the affected shoulder.

To calculate the complication and surgical pitfall rates associated with the reverse prosthesis, we counted the number of patients who experienced at least one intraoperative or postoperative complication and divided this number by the total number of individuals at risk for a complication. To test the hypothesis that the complication rate would be higher in the first patients treated by the

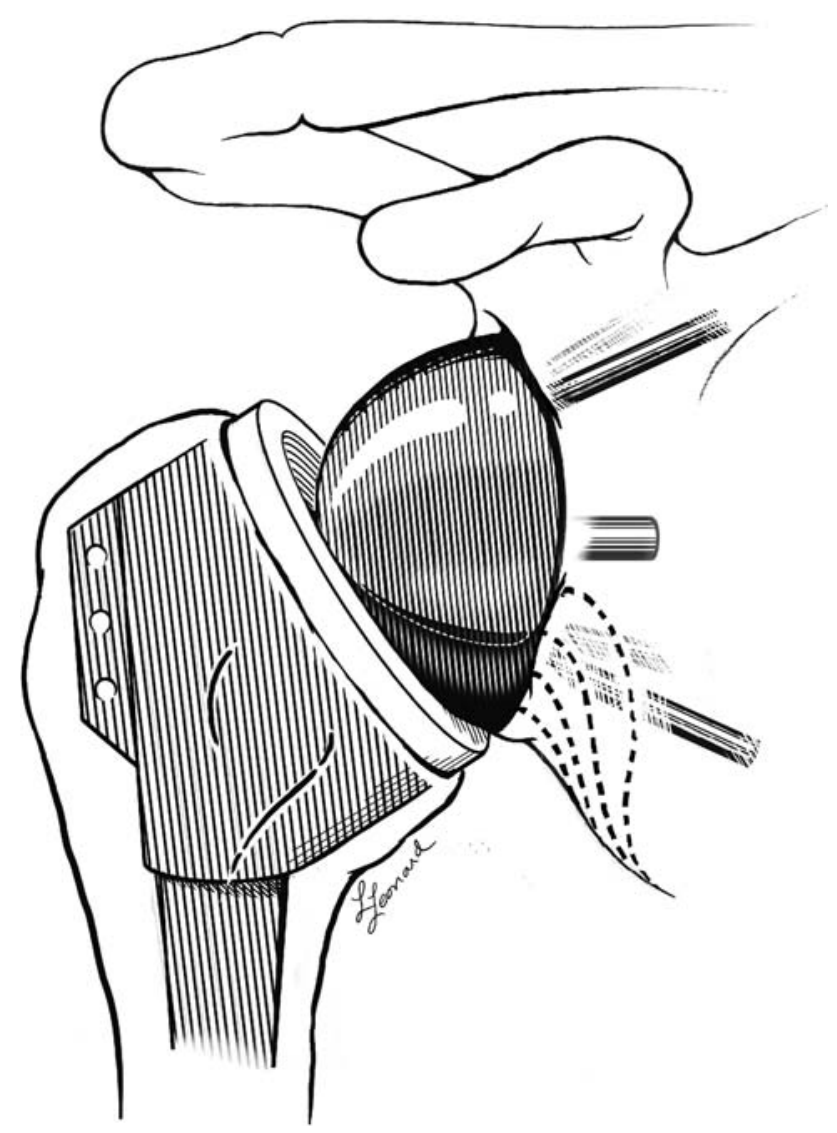

Fig. 3 The Nerot classification (as presented by Valenti et al. [28]) of progressive scapular notching is shown: Grade 0, no notch; Grade 1 (lowest dotted line), notch confined to the scapular pillar; Grade 2 (lower middle dotted line), notch in contact with the lower screw; Grade 3 (upper middle dotted line), notch over the lower screw; Grade 4 (top dotted line), notch extending under the baseplate. (Modified and reprinted with permission and copyright (c) 2005 of The Journal of Bone and Joint Surgery, Inc, from Werner CM, Steinmann PA, Gilbart M, Gerber C. Treatment of painful pseudoparesis due to irreparable rotator cuff dysfunction with the Delta III reverse-balland-socket total shoulder prosthesis. J Bone Joint Surg Am. 2005;87:1476-1486.)

surgeon, the patient sample was divided into two cohorts: early and late experience. We determined the point at which the complication rate decreased (intraoperatively or postoperatively) via an analysis of the running complication rate. This rate was defined as the total number of complications observed divided by the number of patients. The point at which there was a reduction in the running complication rate was considered evidence of proficiency with the surgical technique. A chi square test was used to test the association between experience (early versus later) and complication (intraoperative or postoperative).

We stratified by the independent variable of surgeon experience (early versus late). A similar analysis was used to test the association of risk factors that might contribute to the complication rate: age group ( $\geq 75$ years old), 
gender, and history of previous shoulder surgery. After assessing each variable independently for its influence on the likelihood of a complication, a multivariate model was fit with logistic regression methods to assess all variables simultaneously for their influence on the likelihood of a complication.

From 12 published studies with this reverse prosthesis, we extracted various data about patient population, followup, and complications (Table 1). With this published experience, we constructed $95 \%$ confidence intervals for complication and reoperation rates and compared our experience with that in the literature. For all our analyses, SAS ${ }^{\circledR}$ Version 9.1 (SAS Institute Inc, Cary, NC) was used.

\section{Results}

We observed a higher $(\mathrm{p}=0.037)$ complication rate $(15$ of 20 patients or $75 \%$ ) than the pooled estimate from the literature. In the 12 published articles reporting on 417 patients (Table 1), the mean age at surgery was 62.5 years (pooled SD, 8.3 years), mean followup was 44 months (pooled SD, 14 months), and mean complication rate was $24.7 \%$ (95\% confidence interval, $13.5 \%-35.9 \%$ ).

The observed intraoperative complication rate for the first cohort was greater $(p=0.025)$ than that for the second cohort (Table 2). Individuals in the second cohort were approximately $10 \%$ as likely (odds ratio $=0.11 ; 95 \%$ confidence interval, $0.01-0.84 ; \mathrm{p}=0.033$ ) to have an intraoperative complication as those in the first cohort (Table 2). However, we found similar $(\mathrm{p}=0.361)$ postoperative complication rates between the first and second cohorts (Table 3). For all complications, the number of complications peaked with the seventh patient and then began a steady decline.

After adjusting for age group and gender, individuals in the second cohort were only $7 \%$ as likely to have an intraoperative complication as those in the first cohort

Table 2. Intraoperative complications

\begin{tabular}{lclc}
\hline Complication & $\begin{array}{l}\text { Number in } \\
\text { first cohort }\end{array}$ & $\begin{array}{l}\text { Number in } \\
\text { second cohort }\end{array}$ & $\begin{array}{l}\text { Total } \\
\text { number }\end{array}$ \\
\hline Glenoid fracture & 1 & 1 & 2 \\
Calcar fracture & 1 & 0 & 1 \\
Humeral shaft fracture & 1 & 0 & 1 \\
Medial vault penetration & 3 & 0 & 3 \\
Center glenoid hole too large & 2 & 1 & 2 \\
Center glenoid hole redrilled & 0 & 1 & 2 \\
Poor screw fixation & 5 & 3 & 8 \\
No anterior screw & 2 & 1 & 3 \\
Total complications & 15 & 7 & 22 \\
\hline
\end{tabular}

Table 3. Postoperative complications

\begin{tabular}{lllc}
\hline Complication & $\begin{array}{l}\text { Number in } \\
\text { first cohort }\end{array}$ & $\begin{array}{l}\text { Number in } \\
\text { second cohort }\end{array}$ & $\begin{array}{l}\text { Total } \\
\text { number }\end{array}$ \\
\hline Stitch abscess & 3 & 1 & 4 \\
Superficial wound infection & 0 & 1 & 1 \\
Brachial plexus injury & 1 & 1 & 2 \\
Dislocation & 1 & 1 & 2 \\
Return to operating room & 1 & 1 & 2 \\
Total complications & 6 & 5 & 11 \\
\hline
\end{tabular}

Table 4. Presence of heterotopic ossification on followup radiographs

\begin{tabular}{lll}
\hline $\begin{array}{l}\text { Grade of } \\
\text { heterotopic ossification }\end{array}$ & Number & $\begin{array}{l}\% \text { of affected } \\
\text { patients }\end{array}$ \\
\hline 1 & 6 & 35 \\
2 & 3 & 15 \\
3 & 0 & 0 \\
4 & 0 & 0 \\
\hline
\end{tabular}

(odds ratio $=0.07 ; 95 \%$ confidence interval, 0.01-0.92; $\mathrm{p}=0.043$ ).

Age, gender, and previous shoulder surgery were not risk factors for the development of postoperative complications.

At a minimum of 3 months followup (mean, 9 months) scapular notching was present in 11 patients: Grade 1, three patients; Grade 2, five patients; and Grade 3, three patients. There was no radiographic evidence of fracture or component loosening, dissociation, or dislodgment. At an average followup of 11.5 months (range, 4-19 months), the intraoperative glenoid and humeral fractures appeared to be healing or healed with no dislodgment or migration of the components. Heterotopic ossification was present on the most recent radiographs in nine of 20 patients (average followup, 9 months; range, 3-21 months) (Table 4).

\section{Discussion}

Reverse total shoulder arthroplasty was introduced approximately 20 years ago as a treatment option for patients with symptomatic glenohumeral arthritis and a deficient rotator cuff but was not used extensively until the late 1990s. In numerous series, the reported overall complication rates vary from $0 \%$ to $68 \%$, but these reports do not focus on the intraoperative complications and possible technical explanations under control of the surgeon. Given this variation, we (1) determined the learning curve for the procedure, (2) identified complications and surgical pitfalls, and (3) compared our results with those of similar published series. 
The major limitation of our study was the small sample size. A larger sample size would have provided greater precision for our complication rate estimates. We also would have been able to test for interactions between independent variables. For example, based on the observed rate of postoperative complications, it would have been necessary to follow at least 72 participants in each cohort (total, 144) to achieve an $80 \%$ power analysis to detect a difference between the two groups. However, our study shows that previous surgeon experience did not seem to affect the postoperative complication rate in the first compared with the second 10 patients, so additional study with larger numbers of patients is warranted to evaluate if there is such an effect. Another limitation was the shortterm followup. Without longer-term followup, it is not possible to determine the influence of the surgical pitfalls or the complications on the final clinical result. Because we did not evaluate preoperative or postoperative function or patient satisfaction, the effect of these pitfalls and complications on the final outcome or long-term results is unknown. Our findings should be interpreted in light of two other factors. First, the experience of one surgeon may not be extrapolated to that of other surgeons whose experience levels might vary. Reverse prosthesis surgery is difficult with a high early-experience iatrogenic morbidity even for more experienced shoulder surgeons; although it might be inferred less experienced surgeons would have even more difficulty, we did not address this point. Second, the intraoperative and postoperative complications specific to the reverse prosthesis may be related to the implant designs used and may not apply to other reverse prosthesis systems.

We observed a higher complication rate and wider variety of complications and technical pitfalls associated with the reverse prosthesis than previously reported. We showed there were more complications in the first 10 procedures performed than in the second 10. In our study, the learning curve for our experienced shoulder surgeon appeared to be seven patients, after which the complication rate decreased. These findings are important for any surgeon contemplating or currently performing reverse total shoulder arthroplasty [23]. Comparison of our complication rate with published rates (Table 1) confirms the major source of surgical pitfalls and complications is related to glenoid preparation and baseplate insertion (Table 1). However, few studies focus on intraoperative complications or report surgical pitfalls as potential complications. The number of screws required for adequate fixation of the baseplate is unknown (at least one reverse prosthesis system has no anterior or posterior screws), and screws with poor fixation may not affect long-term results. The deltopectoral approach is associated with a higher instability rate than the superior approach, and this may have had an impact on the complication rate in our study [1].

The type and distribution of complications may have been affected by the number of patients undergoing revision surgery. Complication rates reportedly are higher in patients having revision surgery than in patients with primary arthroplasty [20, 30]. We did not have enough patients with revision of an arthroplasty to a reverse prosthesis to evaluate this variable independent of other variables. However, the only patient with a humeral-side complication in our study did have revision of a conventional shoulder arthroplasty humeral stem.

Our data confirm those of others suggesting most complications occur on the glenoid or baseplate side of the reverse prosthesis (Table 1). Other complications that can occur with glenoid implantation include placement of the prosthesis in the wrong location or orientation. Our rate of scapular notching with short-term followup is similar to rates in other studies with longer followup (Table 5). Recently, Simovitch et al. [24] reported a prosthesisscapular neck angle of $124^{\circ} \pm 19^{\circ}$ predisposed the

Table 5. Reported incidence of scapular notching

\begin{tabular}{|c|c|c|c|c|c|}
\hline Study & $\begin{array}{l}\text { Number of } \\
\text { patients }\end{array}$ & Age $^{*}$ (years) & Prosthesis $^{\dagger}$ & Followup* (months) & $\begin{array}{l}\text { Number with } \\
\text { scapular notching }\end{array}$ \\
\hline Valenti et al. [28] (2001) & 22 & $70(55-87)$ & Delta III & $84(60-108)$ & $19(86 \%)$ \\
\hline Sirveaux et al. [25] (2001) & 80 & $72(58-86)$ & Delta III & $44.5(24-101)$ & $50(65 \%)$ \\
\hline Boulahia et al. [2] (2002) & 16 & $77(66-80)$ & Delta III & $35(24-65)$ & $10(63 \%)$ \\
\hline De Wilde et al. [4] (2003) & 13 & $49(26-68)$ & Delta III & $36(5-120)$ & $4(31 \%)$ \\
\hline Vanhove and Beugnies [29] (2004) & 14 & $71(55-85)$ & Delta III & $31(11-50)$ & $7(50 \%)$ \\
\hline Sirveaux et al. [26] (2004) & 80 & $73(60-86)$ & Delta III & $44(24-97)$ & $49(64 \%)$ \\
\hline Boileau et al. [1] (2005) & 45 & NR & Delta III & $40(24-72)$ & $24(74 \%)$ \\
\hline Werner et al. [30] (2005) & 58 & $68(44-84)$ & Delta III & $38(24-N R)$ & $56(97 \%)$ \\
\hline Frankle et al. [9] (2005) & 60 & $71(34-86)$ & Reverse Shoulder Prosthesis & $33(24-68)$ & $0(0 \%)$ \\
\hline
\end{tabular}

* Values expressed as means, with ranges in parentheses; ${ }^{\dagger}$ prostheses included the Delta III ${ }^{\circledR}$ Reverse Shoulder System (DePuy Orthopaedics, Inc, Warsaw, IN) and the Reverse ${ }^{\mathbb{R}}$ Shoulder Prosthesis (Encore Medical, LP, Austin, TX); NR = not reported. 
prosthesis to notching. We observed scapular notching within the first 3 months after reverse prosthesis implantation in 11 of 20 patients; this may be attributable in part to surgeon inexperience, design of the particular prosthesis used, or improper orientation of the glenoid baseplate on the glenoid. In fact, notching can begin within weeks of implantation of the prosthesis. Because our postoperative radiographs were not obtained with fluoroscopy, we did not analyze baseplate inclination angle and cannot comment on its role in the development of scapular notching in our cohort. Additional study is warranted to determine the relationship between notching during the early postoperative period and subsequent clinical results $[6,24,25,30]$.

We found the existing guides for drilling the central peg of the reverse prosthesis are difficult to control and can contribute to improper drilling of the glenoid baseplate peg hole. Design changes or cannulated systems might help prevent this complication. Complications that we experienced but were not mentioned in the literature included oversizing of the baseplate peg hole when the glenoid bone was soft and penetration of the medial vault of the glenoid by the peg hole drill. Longer followup is necessary to judge the impact of these technical complications.

In our study and in published reports, humeral-side complications were much less common than glenoid baseplate complications (Table 1). With the designs we used, fracture of the proximal humerus can be common if the diameter of the proximal humeral head area is smaller than $36 \mathrm{~mm}$. Propagation of cracks into the proximal humeral metaphyseal area can occur during cementation and implantation of the prosthesis. In two patients, we used cables to prevent fracture of the shaft after implantation. Because these fractures were very proximal in the humeral shaft, it is unlikely they will propagate, but longer-term followup is necessary to assess the clinical significance of this complication.

Numerous studies suggest surgeon experience can affect postoperative clinical results and other outcome variables $[13,14,16-18,27]$. Although one of our goals was to examine the early experience with implantation of the reverse prosthesis, we found the intraoperative complication rate decreased after the first 10 cases. We did not assess the effect of other potentially influential variables, such as skilled operating room personnel, operative time, or case complexity. Most of our intraoperative complications involved baseplate implantation. We found fracture of the glenoid can be minimized by hand reaming the glenoid and accurate placement and orientation of the central peg of the baseplate remains difficult. The effect of compression screws with inadequate purchase on glenoid baseplate fixation is not known. We also found scapular notching is common even during the first 3 months after implantation. Determining the exact impact of these technical and surgical complications on the final clinical result requires additional study. Modifications in implant design and in instrumentation may help to decrease the surgical pitfalls and the intraoperative and postoperative complications we observed.

\section{References}

1. Boileau P, Watkinson DJ, Hatzidakis AM, Balg F. Grammont reverse prosthesis: design, rationale, and biomechanics. $J$ Shoulder Elbow Surg. 2005;14(1 suppl S):147S-161S.

2. Boulahia A, Edwards TB, Walch G, Baratta RV. Early results of a reverse design prosthesis in the treatment of arthritis of the shoulder in elderly patients with a large rotator cuff tear. Orthopedics. 2002;25:129-133.

3. Cook JA, Ramsay CR, Fayers P. Using the literature to quantify the learning curve: a case study. Int J Technol Assess Health Care. 2007;23:255-260.

4. De Wilde L, Sys G, Julien Y, Van Ovost E, Poffyn B, Trouilloud $P$. The reversed Delta shoulder prosthesis in reconstruction of the proximal humerus after tumour resection. Acta Orthop Belg. 2003;69:495-500.

5. De Wilde LF, Plasschaert FS, Audenaert EA, Verdonk RC. Functional recovery after a reverse prosthesis for reconstruction of the proximal humerus in tumor surgery. Clin Orthop Relat Res. 2005;430:156-162.

6. Favard L, Lautmann S, Sirveaux F, Oudet D, Kerjean Y, Huguet D. Hemiarthroplasty versus reverse arthroplasty in the treatment of osteoarthritis with massive rotator cuff tear. In: Walch G, Boileau P, Mole D, eds. Shoulder Prosthesis: Two to Ten Year Follow-up. Montpellier, France: Sauramps Medical; 2001:261268.

7. Fleisch MC, Newton J, Steinmetz I, Whitehair J, Hallum A, Hatch KD. Learning and teaching advanced laparoscopic procedures: do alternating trainees impair a laparoscopic surgeon's learning curve? J Minim Invasive Gynecol. 2007;14:293-299.

8. Frankle M, Levy JC, Pupello D, Siegal S, Saleem A, Mighell M, Vasey M. The Reverse Shoulder Prosthesis for glenohumeral arthritis associated with severe rotator cuff deficiency: a minimum two-year follow-up study of sixty patients: surgical technique. J Bone Joint Surg Am. 2006;88(suppl 1, pt 2):178190.

9. Frankle M, Siegal S, Pupello D, Saleem A, Mighell M, Vasey M. The Reverse Shoulder Prosthesis for glenohumeral arthritis associated with severe rotator cuff deficiency: a minimum twoyear follow-up study of sixty patients. J Bone Joint Surg Am. 2005;87:1697-1705.

10. Grammont P, Trouilloud P, Laffay JP, Deries X. Etude et réalisation d'une nouvelle prothèse d'épaule. Rheumatologie. 1987;39:407-418.

11. Grammont PM, Baulot E. Delta shoulder prosthesis for rotator cuff rupture. Orthopedics. 1993;16:65-68.

12. Guery J, Favard L, Sirveaux F, Oudet D, Mole D, Walch G. Reverse total shoulder arthroplasty: survivorship analysis of eighty replacements followed for five to ten years. J Bone Joint Surg Am. 2006;88:1742-1747.

13. Hammond JW, Queale WS, Kim TK, McFarland EG. Surgeon experience and clinical and economic outcomes for shoulder arthroplasty. J Bone Joint Surg Am. 2003;85:2318-2324.

14. Jain N, Pietrobon R, Hocker S, Guller U, Shankar A, Higgins LD. The relationship between surgeon and hospital volume and outcomes for shoulder arthroplasty. J Bone Joint Surg Am. 2004;86:496-505. 
15. Kjaersgaard-Andersen P, Frich LH, Sojbjerg JO, Sneppen O. Heterotopic bone formation following total shoulder arthroplasty. J Arthroplasty. 1989;4:99-104.

16. Kreder HJ, Deyo RA, Koepsell T, Swiontkowski MF, Kreuter W. Relationship between the volume of total hip replacements performed by providers and the rates of postoperative complications in the state of Washington. J Bone Joint Surg Am. 1997;79:485494.

17. Lavernia CJ. Hemiarthroplasty in hip fracture care: effects of surgical volume on short-term outcome. J Arthroplasty. 1998;13: 774-778.

18. Lavernia CJ, Guzman JF. Relationship of surgical volume to short-term mortality, morbidity, and hospital charges in arthroplasty. J Arthroplasty. 1995;10:133-140.

19. Levy J, Frankle M, Mighell M, Pupello D. The use of the reverse shoulder prosthesis for the treatment of failed hemiarthroplasty for proximal humeral fracture. $J$ Bone Joint Surg Am. 2007;89:292-300.

20. Matsen FA 3rd, Boileau P, Walch G, Gerber C, Bicknell RT. The reverse total shoulder arthroplasty. J Bone Joint Surg Am. 2007;89:660-667.

21. Nwakama AC, Cofield RH, Kavanagh BF, Loehr JF. Semiconstrained total shoulder arthroplasty for glenohumeral arthritis and massive rotator cuff tearing. J Shoulder Elbow Surg. 2000;9:302307.

22. Rittmeister M, Kerschbaumer F. Grammont reverse total shoulder arthroplasty in patients with rheumatoid arthritis and nonreconstructible rotator cuff lesions. J Shoulder Elbow Surg. 2001;10: $17-22$.

23. Rockwood CA Jr. The reverse total shoulder prosthesis: the new kid on the block. J Bone Joint Surg Am. 2007;89:233-235.
24. Simovitch RW, Zumstein MA, Lohri E, Helmy N, Gerber C. Predictors of scapular notching in patients managed with the Delta III reverse total shoulder replacement. J Bone Joint Surg Am. 2007;89:588-600.

25. Sirveaux F, Favard L, Oudet D, Huguet D, Lautman S. Grammont inverted total shoulder arthroplasty in the treatment of glenohumeral osteoarthritis with massive and nonrepairable cuff rupture. In: Walch G, Boileau P, Mole D, eds. Shoulder Prosthesis: Two to Ten Year Follow-up. Montpellier, France: Sauramps Medical; 2001:247-252.

26. Sirveaux F, Favard L, Oudet D, Huquet D, Walch G, Mole D. Grammont inverted total shoulder arthroplasty in the treatment of glenohumeral osteoarthritis with massive rupture of the cuff: results of a multicentre study of 80 shoulders. J Bone Joint Surg Br. 2004;86:388-395.

27. Taylor HD, Dennis DA, Crane HS. Relationship between mortality rates and hospital patient volume for Medicare patients undergoing major orthopaedic surgery of the hip, knee, spine, and femur. J Arthroplasty. 1997;12:235-242.

28. Valenti PH, Boutens D, Nerot C. Delta 3 reversed prosthesis for osteoarthritis with massive rotator cuff tear: long-term results (> 5 years). In: Walch G, Boileau P, Mole D, eds. Shoulder Prosthesis: Two to Ten Year Follow-up. Montpellier, France: Sauramps Medical; 2001:253-259.

29. Vanhove B, Beugnies A. Grammont's reverse shoulder prosthesis for rotator cuff arthropathy: a retrospective study of 32 cases. Acta Orthop Belg. 2004;70:219-225.

30. Werner CM, Steinmann PA, Gilbart M, Gerber C. Treatment of painful pseudoparesis due to irreparable rotator cuff dysfunction with the Delta III reverse-ball-and-socket total shoulder prosthesis. J Bone Joint Surg Am. 2005;87:1476-1486. 